\title{
Aspectos biológicos de Chrysoperla raimundoi Freitas \& Penny (Neuroptera, Chrysopidae)
}

\author{
Taís Carmona Lavagnini ${ }^{1}$, Sérgio de Freitas ${ }^{1} \&$ Antônio Lourenço Bezerra ${ }^{2}$
}

\begin{abstract}
'Departamento de Fitossanidade, Faculdade de Ciências Agrárias e Veterinárias, UNESP, Via de acesso Prof. Paulo Donato Castellane, s/n, 14884-900 Jaboticabal-SP, Brasil. taisc_lavagnini@yahoo.com.br; serfre@ fcav.unesp.br

${ }^{2}$ Departamento de Fitossanidade, Universidade Federal Rural de Pernambuco, UFRPE, Bairro: Dois Irmãos, 52171-900 Recife-PE, Brasil. alourencob@ hotmail.com
\end{abstract}

\begin{abstract}
Biological aspects of Chrysoperla raimundoi Freitas \& Penny (Neuroptera, Chrysopidae). The green lacewings are important predators and are found in several agroecosystems. The temperature is one of the decisive factors for the development of insects, and there is an acceptable variation gradient for each species. Therefore, the purpose of this study was not only to check the influence of different temperatures on the embryonic and postembryonic development of Chrysoperla raimundoi, as well as check the consequences in the different generations. The immature stages were reared at four different environmental conditions, three of them with constant temperatures $\left(19,0,25,0\right.$ e $\left.31,0^{\circ} \mathrm{C}\right)$ and the fourth rearing place was external to the laboratory without control of the abiotic factors. The duration of the embryonic period, first, second and third larval instar, pupal stage, and from egg to adult were evaluated; also the survival rate of each instar and stage and the emergence rate were calculated. The embryonic and post embryonic development differed between the generations $\mathrm{F}_{2}$ and $\mathrm{F}_{4}$. Also, the influence of different temperatures for the generation $\mathrm{F}_{2}$ among individuals created at $31,0^{\circ} \mathrm{C}$ showed reduction on the development rate regarding the other two environments. It was possible to observe that the generation $\mathrm{F}_{4}$ has its development influenced by different temperatures, and the $19,0^{\circ} \mathrm{C}$ temperature prolonged its development.
\end{abstract}

KEYWORDS. Green lacewing; insect development; life cycle; thermal requirements.

\begin{abstract}
RESUMO. Aspectos biológicos de Chrysoperla raimundoi Freitas \& Penny (Neuroptera, Chrysopidae). Os crisopídeos são importantes predadores e são encontrados em diversos agroecossistemas. A temperatura é um dos fatores determinantes para o desenvolvimento dos insetos, sendo que existe um gradiente de variação aceitável para cada espécie. Portanto, objetivou-se com este estudo verificar a influência de diferentes temperaturas sobre o desenvolvimento embrionário e pós-embrionário de $C$. raimundoi assim como verificar as consequiências nas diferentes gerações. As fases jovens foram criadas em três ambientes com temperaturas constantes de $19,0,25,0$ e $31,0^{\circ} \mathrm{C}$ e em um ambiente externo sem controle dos fatores abióticos. Foram observados a duração do período embrionário, do primeiro, segundo e terceiro ínstares, a duração do período larval, pupal e ovo-adulto, assim como, a sobrevivência em cada estágio, estádio e a porcentagem de emergência. $\mathrm{O}$ desenvolvimento embrionário e pós-embrionário diferiu entre as gerações $\mathrm{F}_{2}$ e $\mathrm{F}_{4}$. Quanto à influência de diferentes temperaturas para a geração $\mathrm{F}_{2}$, os indivíduos criados a $31,0^{\circ} \mathrm{C}$ demonstraram redução na taxa de desenvolvimento com relação aos outros dois ambientes. Para a geração $\mathrm{F}_{4}$, observou-se que as diferentes temperaturas influenciaram no desenvolvimento, sendo que a $19,0^{\circ} \mathrm{C}$ houve prolongamento do mesmo.
\end{abstract}

PALAVRAS-CHAVE. Ciclo de vida; crisopídeo; desenvolvimento; exigências térmicas.

Os crisopídeos são importantes predadores presentes em diversos agroecossistemas. Os adultos possuem hábitos diferentes em relação às formas larvais e essa característica lhes confere vantagem, pois as larvas e os adultos exploram habitats diferentes (Freitas 2002), reduzindo a competição intraespecífica.

São insetos capazes de se alimentar de vários artrópodespraga como pulgões, cochonilhas, ácaros, mosca-branca, psilídeos, ovos de lepidópteros e coleópteros (Freitas 2002). Durante o período larval, uma larva de Chrysoperla externa (Hagen, 1861) se alimenta de 567,4 ovos de Diatraea saccharalis (Fabricius, 1794) (Lepidoptera, Crambidae); 930,2 ovos de Sitotroga cerealella (Oliver,1819) (Lepidoptera, Gelechiidae) e 1553 ovos de Anagasta kuehniella (Zeller, 1879)
(Lepidoptera, Pyralidae) (Murata et al. 2006). Larvas de $C$. externa são capazes de consumir diariamente durante o terceiro ínstar cerca de 41,3 de ninfas de Uroleucon ambrosiae (Thomas, 1878) (Hemiptera, Aphididae), importante vetor do vírus-do-mosaico-da-alface, quando oferecidas na densidade de 50 indivíduos (Auad et al. 2003).

A temperatura é um dos fatores determinantes para o desenvolvimento dos insetos, existindo um gradiente de variação aceitável para cada espécie, necessitando de uma faixa de temperatura ideal para o seu desenvolvimento. Essa faixa está relacionada com aquela prevalente nos locais onde o inseto vive, entretanto, populações diferentes podem ter exigências térmicas diferentes (Andrewartha \& Birch 1954).

Fonseca et al. (2001) observaram que em ambientes com 
temperatura constante de 15,$0 ; 24,0$ e $30,0^{\circ} \mathrm{C}$ a duração do período larval para C. externa foi de 36,5; 11,0 e 9,4 dias respectivamente. Para Ceraeochrysa paraguaria (Navás, 1919) observou-se que a duração do período larval em ambientes com temperaturas constantes de 15,0 e $25,0^{\circ} \mathrm{C}$ foi de 57,0 e 12,5 dias respectivamente (Pessoa et al. 2004b).

Conhecer as exigências térmicas de $C$. raimundoi Freitas \& Penny, 2001 é de fundamental importância para determinar temperaturas de criação e armazenamento desses insetos nas fases imaturas, assim como variar a temperatura para sincronismo de desenvolvimento. A temperatura base consiste na temperatura mais baixa suportada pelo inseto em que o metabolismo seja reduzido sem que haja a morte deles, sendo importante para determinar temperaturas de armazenamento durante o desenvolvimento. A constante térmica é importante para se determinar a quantidade de quilocalorias necessárias para o desenvolvimento do inseto na temperatura ambiente.

Para C. externa foi observado que a temperatura base varia de 9,5 a 11,5 e a constante térmica varia de 36,0 a 290,2 grausdia entre os estágios e estádios (Figueira et al. 2000).

Em razão da descrição recente do crisopídeo C. raimundoi, este trabalho teve o objetivo de avaliar a influência de diferentes temperaturas sobre o desenvolvimento embrionário e pós-embrionário desse crisopídeo em duas gerações sucessivas, além de determinar as exigências térmicas dessa espécie.

\section{MATERIALE MÉTODOS}

O presente estudo foi conduzido no Laboratório de Biossistemática e Biologia Molecular de Crisopídeos (LBBMC) da Faculdade de Ciências Agrárias e Veterinárias, UNESP, Campus Jaboticabal, São Paulo. O experimento foi realizado em três ambientes, três repetições cada, sendo que em cada repetição havia 50 indivíduos de C. raimundoi da geração $\mathrm{F}_{2}$. Para dois ambientes foram utilizadas salas climatizadas com fotoperíodo de 12 horas, umidade relativa de $70 \pm 10 \%$ e temperaturas constantes de 25,0 e $31,0^{\circ} \mathrm{C}$. Como ambiente externo, sem controle dos fatores abióticos, foi utilizado uma área coberta fora do laboratório, o fotoperíodo foi natural.

Os indivíduos utilizados na experimentação foram obtidos a partir da criação de manutenção do LBBMC. Os ovos foram individualizados em recipientes de vidro transparente e incolor de $1,5 \mathrm{~cm}$ de diâmetro por $2,5 \mathrm{~cm}$ de altura vedados com filme plástico. As larvas eclodidas foram alimentadas com ovos de $S$. cereallella. Os ovos foram oferecidos aderidos em cartelas de papel com as seguintes dimensões $0,5 \mathrm{~cm} \times 0,5 \mathrm{~cm}$ para $\mathrm{o}$ primeiro ínstar, $0,5 \mathrm{~cm} \times 1,0 \mathrm{~cm}$ para o segundo ínstar e $0,5 \mathrm{~cm} \times$ $1,5 \mathrm{~cm}$ para o terceiro ínstar.

Para análise da influência de uma temperatura menor que aquela considerada ideal para criação de insetos $\left(25,0^{\circ} \mathrm{C}\right)$, ovos e larvas de $C$. raimundoi (geração $\mathrm{F}_{4}$ ) foram submetidos a dois ambientes, ambos com temperaturas constantes (19,0 e $25,0^{\circ} \mathrm{C}$ ), fotoperíodo de 12 horas e umidade relativa de $70 \pm 10$ $\%$. A metodologia de criação foi a mesma utilizada para os indivíduos da geração $\mathrm{F}_{2}$.
Para verificar a diferença entre as gerações, foram utilizados os dados obtidos a partir dos indivíduos submetidos ao ambiente a $25^{\circ} \mathrm{C}$ das gerações $\mathrm{F}_{2}$ e $\mathrm{F}_{4}$.

Em todos os experimentos foram observados a duração do período embrionário, do primeiro, segundo e terceiro ínstares, a duração do período larval, pupal e ovo-adulto, assim como a sobrevivência em cada estágio e estádio e a porcentagem de emergência. Os dados obtidos foram analisados pelo Teste de Tukey a 5\% de probabilidade, para verificar a significância entre os dados. As análises foram realizadas através do software Estat (ESTAT 1992). Para o cálculo do limiar térmico inferior $(\mathrm{Tb})$ e constante térmica $(\mathrm{K})$ empregou-se a metodologia de Haddad \& Parra (1984).

\section{RESULTADOSEDISCUSSÃO}

A temperatura média, para o ambiente externo, obtida foi $25,8 \pm 0,4^{\circ} \mathrm{C}$, sendo a máxima $31,7^{\circ} \mathrm{C}$ e a mínima $19,8^{\circ} \mathrm{C}$; a umidade relativa média foi de 74,2\%, máxima de $96,0 \%$ e mínima de $52,5 \%$, o fotoperíodo foi natural.

Constatou-se que o período embrionário para os ovos da geração $\mathrm{F}_{2}$ não diferiu quando foram mantidos em local com flutuação da temperatura $\left(25,8^{\circ} \mathrm{C}\right)$ e à temperatura de $25,0^{\circ} \mathrm{C}$. A temperatura mais elevada promoveu a redução de $32 \%$ na duração do período embrionário quando comparado as outras condições térmicas, e a porcentagem de eclosão foi de $83,3 \%$ (Tabela I).

As durações do primeiro e segundo ínstares não diferiram significativamente entre o ambiente com flutuação da temperatura e a $25,0^{\circ} \mathrm{C}$. Contudo, diferiram significativamente daqueles encontrados para as larvas criadas a $31,0^{\circ} \mathrm{C}$. As porcentagens de sobrevivência no primeiro e segundo ínstares foram superiores a $95,1 \%$, foi observado $100 \%$ de sobrevivência para larvas de segundo ínstar submetidas ao ambiente externo (Tabela I).

As larvas criadas nos três ambientes não apresentaram diferenças significativas quanto à duração do terceiro ínstar, evidenciando que a elevação ou flutuação da temperatura diária não alteraram esse parâmetro bem como a porcentagem de sobrevivência. As larvas de C. raimundoi mantidas em ambiente onde houve flutuação diária da temperatura, umidade relativa do ar e fotoperíodo, sofreram a ação da variação de amplitude da temperatura.

Larvas submetidas a $31,0^{\circ} \mathrm{C}$ apresentaram redução do período larval que diferiu dos demais ambientes. O período larval foi $18,0 \%$ menor do que a $25,0^{\circ} \mathrm{C}$ e $15,5 \%$ menor do que no ambiente com flutuação da temperatura. Contudo, sua redução não promoveu efeitos negativos à sobrevivência neste período, apresentando porcentagem de sobrevivência, de $91,8 \%$. Os valores médios para o período larval observados para o ambiente com flutuação da temperatura e $25,0^{\circ} \mathrm{C}$ não diferiram entre si significativamente, variando apenas $3 \%$ e as porcentagens de sobrevivência para estes ambientes foram superiores a $92 \%$.

Observou-se redução na duração do período pupal na temperatura de $31,0^{\circ} \mathrm{C}$, diferindo significativamente das demais. 

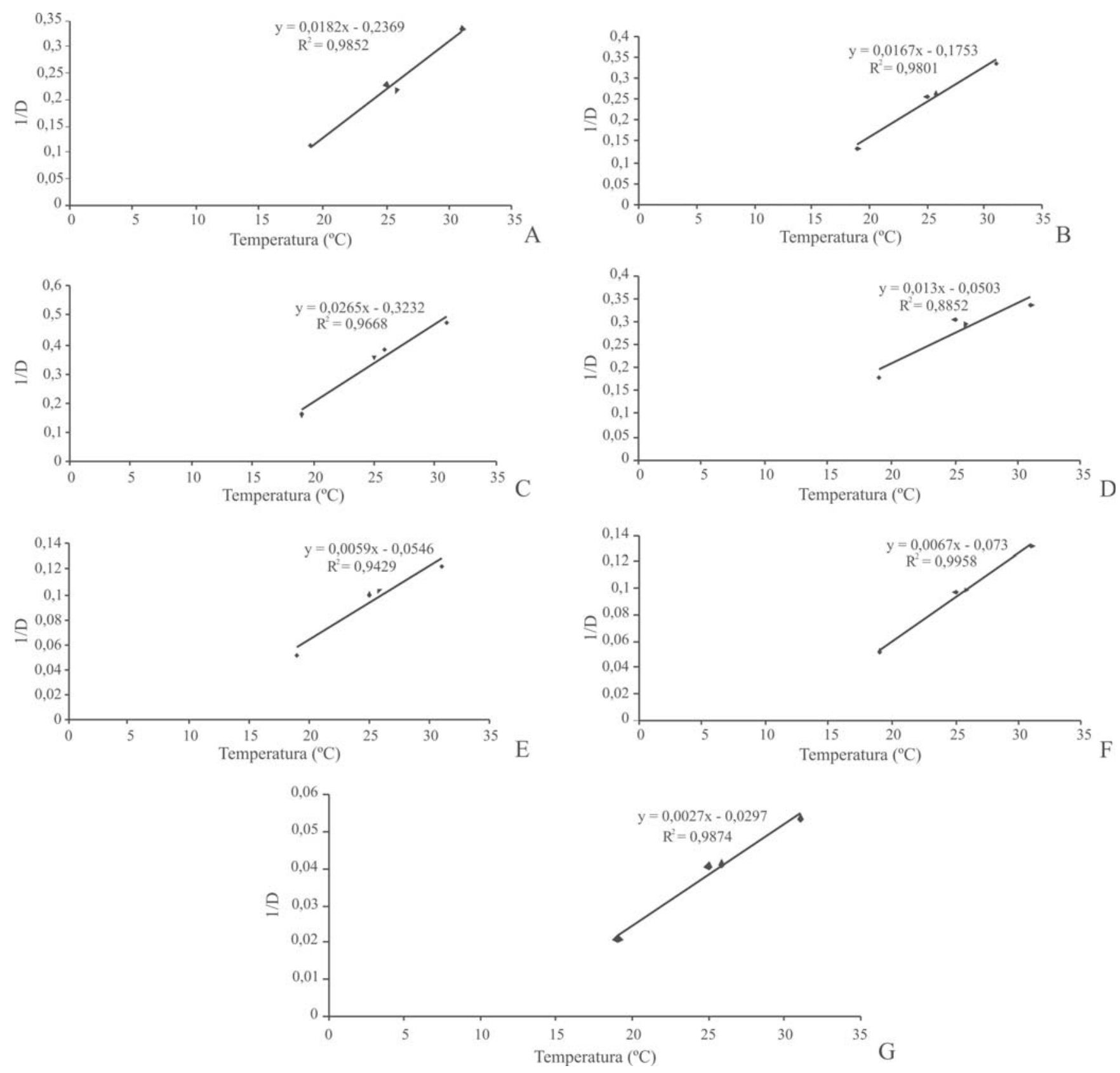

Fig. 1. Curvas de regressão ajustadas para a interação entre temperatura e a duração do desenvolvimento de Chrysoperla raimundoi. A: período embrionário, B: primeiro ínstar; C: segundo ínstar; D: terceiro ínstar; E: período larval; F: período pupal; G: período ovo-adulto.

A flutuação diária da temperatura não influenciou de forma negativa a duração deste período com relação a $25,0^{\circ} \mathrm{C}$. As porcentagens de sobrevivência foram de $88,9,86,4$ e $97,5 \%$.

A duração do período ovo-adulto para indivíduos mantidos a $25,0^{\circ} \mathrm{C}$ e aquelas em temperatura ambiente, não diferiram significativamente. A duração desse período foi menor para larvas submetidas ao ambiente a $31,0^{\circ} \mathrm{C}$, diferindo significativamente das demais condições climáticas avaliadas (Tabela I). Em relação à porcentagem de emergência (Tabela I) diferiu significativamente entre todas as temperaturas avaliadas. Na temperatura de $31,0^{\circ} \mathrm{C}$ observou-se que ao findar o período pupal houve a emergência de sub-imagos, mas estes não concluíram o desenvolvimento para formação de um adulto normal. Esses sub-imagos após alguns dias escureciam e morriam, justificando assim a porcentagem de emergência baixa $(15,2 \%)$.

Apesar da semelhança entre os valores médios obtidos para o ambiente a $25,0^{\circ} \mathrm{C}$ e com flutuação da temperatura quanto à duração do desenvolvimento embrionário e pós-embrionário, o ambiente com flutuação da temperatura proporcionou a obtenção de maior número de adultos que o ambiente a $25,0^{\circ} \mathrm{C}$ (Tabela I). Esse fato é importante, tendo em vista que foi 
Tabela I. Duração em dias (D) do período embrionário, dos ínstares, dos períodos larval, pupal e ovo-adulto; porcentagem (\%) de sobrevivência em cada estádio e estágio e porcentagem de emergência para Chrysoperla raimundoi nas temperaturas $25,0,25,8$ e $31^{\circ} \mathrm{C}$ para a geração $\mathrm{F}_{2}$ e nas temperaturas 19 e $25^{\circ} \mathrm{C}$ para a geração $\mathrm{F}_{4}$.

\begin{tabular}{|c|c|c|c|c|c|c|c|c|c|c|}
\hline & \multicolumn{6}{|c|}{ Geração $F_{2}$} & \multicolumn{4}{|c|}{ Geração $F_{4}$} \\
\hline & \multicolumn{2}{|c|}{$25,0^{\circ} \mathrm{C}$} & \multicolumn{2}{|c|}{$25,8^{\circ} \mathrm{C}$} & \multicolumn{2}{|c|}{$31,0^{\circ} \mathrm{C}$} & \multicolumn{2}{|c|}{$19,0^{\circ} \mathrm{C}$} & \multicolumn{2}{|c|}{$25,0^{\circ} \mathrm{C}$} \\
\hline & $\mathrm{D}$ & $\%$ & $\mathrm{D}$ & $\%$ & $\mathrm{D}$ & $\%$ & $\mathrm{D}$ & $\%$ & D & $\%$ \\
\hline PE & $4,4 \mathrm{a}$ & 85,3 & $4,6 \mathrm{a}$ & 83,3 & $3,0 \mathrm{~b}$ & 89,3 & $8,9 \mathrm{a}$ & 76,0 & $4,5 \mathrm{~b}$ & 80,7 \\
\hline I1 & $3,9 \mathrm{a}$ & 95,1 & $3,8 \mathrm{a}$ & 98,5 & $3,0 \mathrm{~b}$ & 96,9 & $7,5 \mathrm{a}$ & 92,4 & $4,6 \mathrm{~b}$ & 89,3 \\
\hline $\mathrm{I} 2$ & $2,8 \mathrm{a}$ & 99,2 & $2,6 \mathrm{a}$ & 100,0 & $2,1 \mathrm{~b}$ & 95,6 & $6,2 \mathrm{a}$ & 97,2 & $2,5 \mathrm{~b}$ & 99,1 \\
\hline I3 & $3,3 \mathrm{a}$ & 98,4 & $3,4 \mathrm{a}$ & 98,3 & $3,0 \mathrm{a}$ & 100,0 & $5,6 \mathrm{a}$ & 100,0 & $4,0 \mathrm{~b}$ & 100,0 \\
\hline PL & $10,0 \mathrm{a}$ & 92,8 & $9,7 \mathrm{a}$ & 96,8 & $8,2 \mathrm{~b}$ & 91,8 & $19,3 \mathrm{a}$ & 89,7 & $11,1 \mathrm{~b}$ & 88,4 \\
\hline $\mathrm{PP}$ & $10,4 \mathrm{a}$ & 88,9 & $10,1 \mathrm{a}$ & 97,5 & $7,6 \mathrm{~b}$ & 86,4 & $19,3 \mathrm{a}$ & 95,3 & $9,8 \mathrm{~b}$ & 98,1 \\
\hline POA & $24,8 \mathrm{a}$ & - & $24,4 \mathrm{a}$ & - & $18,7 \mathrm{~b}$ & - & $47,9 \mathrm{a}$ & - & $25,4 \mathrm{~b}$ & - \\
\hline PEM & - & $60,4 \mathrm{~b}$ & - & $93,5 \mathrm{a}$ & - & $15,2 \mathrm{c}$ & - & $76,3 \mathrm{a}$ & - & $78,4 \mathrm{a}$ \\
\hline
\end{tabular}

*Médias seguidas de mesma letra na linha não são significativamente diferentes pelo teste Tukey a 5\% de probabilidade.

$\mathrm{PE}$ = período embrionário; $\mathrm{I} 1=1^{\circ}$ ínstar; $\mathrm{I} 2=2^{\circ}$ ínstar; $\mathrm{I} 3=3^{\circ}$ ínstar; $\mathrm{PL}=$ período larval; $\mathrm{PP}=$ período pupal; POA = período ovo-adulto; PEM $=$ porcentagem de emergência.

possível obter maior número de indivíduos sem o controle de temperatura. Isso evidencia que seria possível fazer a criação de C. raimundoi sem a utilização de condicionadores de ar para controle da temperatura, reduzindo assim os custos da biofábrica, pelo menos durante a estação do ano (verão) em que os experimentos foram conduzidos. Entretanto, é importante ressaltar que os dados obtidos são exclusivos para a população utilizada na experimentação, uma vez que cada população possui exigências térmicas próprias (Andrewartha $\&$ Birch 1954).

A temperatura de $31,0^{\circ} \mathrm{C}$ promoveu redução na duração do período ovo-adulto (Tabela I), diferindo significativamente dos outros dois ambientes. A duração do período ovo-adulto foi $25,0 \%$ menor do que a $25,0^{\circ} \mathrm{Ce} 23,4 \%$ menor do que no ambiente com flutuação da temperatura. $\mathrm{O}$ ambiente a $31,0^{\circ} \mathrm{C}$ proporcionou o rápido desenvolvimento e a porcentagem de emergência (Tabela I) observada para este ambiente foi muito baixa, cerca de $15,2 \%$. Tal ambiente de criação poderia ser utilizado por biofábricas para acelerar a produção do inimigo natural quando a necessidade de sua liberação em campo fosse alta. Contudo, deve-se considerar o baixo número de adultos emergidos. A porcentagem de emergência observada para este ambiente diferiu significativamente dos demais.

Para as biofábricas é interessante que elas mantenham o menor número de unidades de criação de adultos ovipositando, sendo que em cada unidade de criação haja o maior número de adultos possível. Assim, a manutenção da criação torna-se mais dinâmica, pois o trabalho será feito com maior agilidade obtendo-se elevada quantidade de ovos que serão liberados em campo. A comercialização efetiva de agentes para controle biológico depende da habilidade das biofábricas em produzir e comercializar lucrativamente inimigos naturais altamente confiáveis e relativamente baratos (Tauber et al. 2000). Desta forma, os crisopídeos podem ser utilizados em programas de manejo integrado de pragas fazendo-se a liberação periódica de ovos embrionados (Tauber et al. 2000).

Experimentos realizados por Pessoa et al. (2004a) mostraram que Ceraeochrysa cincta (Schneider, 1851), quando submetida a elevadas temperaturas $\left(31,0^{\circ} \mathrm{C}\right)$, tem a duração do seu período pós-embrionário reduzido, de 24 a $46 \%$ dependendo do estádio e estágio considerado. No presente estudo, C. raimundoi também apresentou redução na duração do seu desenvolvimento pós-embrionário, quando submetida ao ambiente a $31,0^{\circ} \mathrm{C}$. Entretanto, Pessoa et al. (2004a) observaram baixa viabilidade durante o período larval, 49,7\%, para $C$. cincta, enquanto que no presente estudo, a viabilidade para larvas de $C$. raimundoi durante o período larval foi elevada, cerca de $91,8 \%$.

Para as fases jovens de C. externa, mantidas em ambientes com diferentes temperaturas, constatou-se que o período de desenvolvimento aumentou em função da elevação da temperatura, desta forma o período ovo-adulto também era reduzido. Comparando-se as durações do período larval entre os ambientes de criação com temperaturas de 18,0 e $30,0^{\circ} \mathrm{C}$ observa-se redução de 60,0\% em relação ao primeiro (Figueira et al. 2000). Entretanto, para C. raimundoi não foi observada a redução acentuada, $18,0 \%$, no período larval como ocorreu para C. externa (Figueira et al. 2000). Isso ocorreu provavelmente porque a amplitude de variação foi das temperaturas foi de $12,0^{\circ} \mathrm{C}$ usada por Figueira et al. (2000) não foi a mesma usada neste experimento $\left(6,0^{\circ} \mathrm{C}, 25,0\right.$ e $\left.31,0^{\circ} \mathrm{C}\right)$.

A temperatura base $(\mathrm{Tb})$ e a constante térmica $(\mathrm{K})$ para os períodos embrionário, larval, pupal, ovo-adulto e os três ínstares apresentaram variações. A temperatura base mais baixa foi de $3,9^{\circ} \mathrm{C}$ para o terceiro ínstar e a mais alta foi de $13,0^{\circ} \mathrm{C}$ para o período embrionário. A constante térmica variou entre 37,7 e 370,4GD (Tabela II e Figura 1). A interação da temperatura com a duração das fases de desenvolvimento de C. raimundoi, bem como as equações lineares e os valores para $\mathrm{R}^{2}$ estão apresentadas na Figura 1. Os coeficientes $\mathrm{R}^{2}$ de todas as interações evidenciaram alta significância, de 0,8852 a 0,9958, entre os fatores duração do desenvolvimento e temperatura do ambiente.

$\mathrm{O}$ uso de baixas temperaturas como forma de diminuir a taxa de metabolismo e desenvolvimento tem sido muito empregada em experimentos com armazenamento de insetos 
(Tulisalo 1984). Contudo, para que sejam armazenados ovos, larvas e pupas, é preciso determinar o limiar térmico inferior (temperatura base). Assim, o metabolismo será reduzido sem que haja a morte do inseto, podendo ser utilizada esta temperatura para armazenamento (Parra 2001).

A partir dos resultados obtidos quanto às exigências térmicas (Tabela II) conclui-se que o armazenamento dos ovos de $C$. raimundoi pode ser feito a partir da temperatura de $13,0^{\circ} \mathrm{C}$ sem que haja eclosão das larvas. Larvas de primeiro, segundo e terceiro ínstares suportam temperatura mínima de criação de 10,$5 ; 12,2$ e $3,9^{\circ} \mathrm{C}$ sem que haja morte dos indivíduos. $\mathrm{O}$ armazenamento de pupas também pode ser realizado desde que seja feito a partir da temperatura de $10,9^{\circ} \mathrm{C}$.

Estudos realizados quanto ao armazenamento de ovos constataram que para Ceraeochrysa cubana (Hagen, 1861) e Ceraeochrysa smithi (Navás, 1914) o período máximo de armazenamento foi de 14 dias a $15,6^{\circ} \mathrm{C}$ (López-Arroyo et al. 2000), para Chrysoperla carnea (Stephens, 1836) foi possível armazená-los por até 20 dias a $8,0^{\circ} \mathrm{C}$ sem perda da viabilidade (Osman \& Selman 1993) e para C. externa pode-se armazenar os ovos a $12,0^{\circ} \mathrm{C}$ por mais de 16 dias e a $9,0^{\circ} \mathrm{C}$ por mais de 10 dias com viabilidade superior a 50,0\% (Saini, 1997).

Os valores obtidos para a constante térmica (Tabela II) também são de extrema importância. Pode-se obter a temperatura de criação ideal para que o desenvolvimento do inseto ocorra no período desejado. Ou também conhecer quanto tempo durará determinado estágio de desenvolvimento do inseto quando se tem uma determinada temperatura de criação desejada.

Influencia da temperatura sobre o desenvolvimento embrionário e pós-embrionário para a geração $\mathrm{F}_{4}$. Os indivíduos da geração $\mathrm{F}_{4}$ sofreram a influência das temperaturas quanto à duração do período embrionário e pós-embrionário (Tabela I).

A temperatura mais baixa $\left(19,0^{\circ} \mathrm{C}\right)$ promoveu a redução da porcentagem do desenvolvimento do embrião e, portanto a duração do período embrionário foi maior do que a $25,0^{\circ} \mathrm{C}$. Houve diferença significativa entre as durações do período embrionário nas duas temperaturas, sendo que no ambiente a $19,0^{\circ} \mathrm{C}$ este período foi $97,7 \%$ maior do que a $25,0^{\circ} \mathrm{C}$. As porcentagens de eclosão foram superiores a 76,0\% sendo que este valor foi maior para os ovos submetidos a $25,0^{\circ} \mathrm{C}$ (Tabela I).

As durações do primeiro, segundo e terceiro ínstares foram mais longas para as larvas criadas a $19,0^{\circ} \mathrm{C}$ do que para aquelas mantidas a $25,0^{\circ} \mathrm{C}$. As variações nas durações dos ínstares entre os dois ambientes diferiram significativamente em todos os casos, sendo que o primeiro, segundo e terceiro ínstares foram $63,0,148,0$ e 40,0\% respectivamente maiores do que a $25,0^{\circ} \mathrm{C}$ (Tabela I). As porcentagens de sobrevivência para os três ínstares foram superiores a 89,3\%, sendo que para o terceiro ínstar foi observado $100,0 \%$ de sobrevivência para os dois ambientes de criação.

Em decorrência do prolongamento da duração dos ínstares a duração do período larval também foi mais longa para os indivíduos submetidos ao ambiente à $19,0^{\circ} \mathrm{C}$. Houve diferença significativa entre os valores médios obtidos para ambos os
Tabela II. Temperatura base $(\mathrm{Tb})$ e constante térmica $(\mathrm{K})$ para as fases de desenvolvimento de Chrysoperla raimundoi.

\begin{tabular}{ccc}
\hline Fases do desenvolvimento & $\mathrm{Tb}\left({ }^{\circ} \mathrm{C}\right)$ & $\mathrm{K}(\mathrm{GD})$ \\
\hline Período embrionário & 13,0 & 54,9 \\
$1^{\circ}$ ínstar & 10,5 & 59,9 \\
$2^{\circ}$ ínstar & 12,2 & 37,7 \\
$3^{\circ}$ ínstar & 3,9 & 76,9 \\
Período larval & 9,3 & 169,5 \\
Período pupal & 10,9 & 149,3 \\
Período ovo-adulto & 11,0 & 370,4 \\
\hline
\end{tabular}

ambientes. A duração deste período à $19,0^{\circ} \mathrm{C}$ foi $73,9 \%$ maior do que à $25,0^{\circ} \mathrm{C}$ e as porcentagens de sobrevivência foram superiores a $88,4 \%$.

A duração do período pupal foi $97,0 \%$ maior para os indivíduos criados a $19,0^{\circ} \mathrm{C}$ do que para aqueles submetidos ao ambiente a $25,0^{\circ} \mathrm{C}$. Estes valores médios observados diferiram significativamente entre si e as porcentagens de sobrevivência foram superiores a 95,3\% (Tabela I).

A redução da porcentagem de desenvolvimento provocada pela baixa temperatura $\left(19,0^{\circ} \mathrm{C}\right)$ de criação alongou o período ovo-adulto (Tabela I). O valor médio obtido neste ambiente foi $88,6 \%$ maior do que o obtido a $25,0^{\circ} \mathrm{C}$, houve diferença significativa entre eles. Isto evidencia que a temperatura $19,0^{\circ} \mathrm{C}$ pode ser utilizada pelas biofábricas para reduzir a taxa de desenvolvimento quando a necessidade de liberação em campo for baixa. Assim, os custos com a manutenção da criação podem ser reduzidos, pois o tempo para obtenção dos adultos será mais longo.

A utilização desta temperatura pelas biofábricas pode ser feita para prolongar o desenvolvimento das fases jovens sem comprometimento no número de adultos obtidos, pois as porcentagens de emergência (Tabela I) não diferiram significativamente entre os ambientes. Portanto, a obtenção de adultos para manutenção da criação não será prejudicada, uma vez que os valores obtidos no ambiente à $19,0^{\circ} \mathrm{C}$ estão próximos daqueles obtidos à $25,0^{\circ} \mathrm{C}$, que é considerada a temperatura ideal para criação de insetos (Canard \& Principi 1984).

Variações na duração do desenvolvimento embrionário e pós-embrionário nas diferentes gerações. A duração do período embrionário não apresentou diferenças entre as duas gerações estudadas, não diferindo significativamente, variando apenas 2,3\%. A sobrevivência neste período foi ligeiramente maior na geração $\mathrm{F}_{2}$ (Tabela I).

Não foram observadas diferenças significativas quanto à duração do primeiro e do segundo ínstares entre as duas gerações. Houve maior sobrevivência nesses estádios na geração $\mathrm{F}_{2}$.

Para a geração $\mathrm{F}_{4}$ obteve-se duração do terceiro ínstar mais longa do que na geração $\mathrm{F}_{2}$, diferindo significativamente. Foi observado $100,0 \%$ de sobrevivência para este ínstar na geração $\mathrm{F}_{4}$ (Tabela I).

Apesar de não ter sido observado diferenças significativas 
na duração dos ínstares, com exceção do terceiro, a duração do período larval diferiu significativamente entre as duas gerações. Este foi mais longo na geração $\mathrm{F}_{4}$ com ligeira queda na sobrevivência (4,8\%). A duração do período pupal também diferiu significativamente entre estas gerações, a variação foi de $5,8 \%$, as porcentagens de sobrevivência foram superiores a $88,9 \%$ (Tabela I).

A partir dos resultados obtidos quanto à duração dos períodos larval e pupal, pode-se inferir que a endogamia, resultante dos acasalamentos, pode influenciar na duração destes períodos devido a redução na variabilidade genética, haja visto que seus valores médios diferiram significativamente entre si. Contudo, a duração do período ovo-adulto (Tabela I) não diferiu significativamente entre as gerações.

As porcentagens de emergência (Tabela I) não diferiram significativamente entre as gerações. $\mathrm{O}$ fato da geração $\mathrm{F}_{4}$ ser derivada da geração $\mathrm{F}_{2}$ pode ter proporcionado a redução da variabilidade genética, entretanto este fato não interferiu na sobrevivência das pupas.

Estudos utilizando quatro gerações sucessivas de $C$. cubana demonstraram que houve variação na duração das fases imaturas e a viabilidade dessas fases não foi afetada, com exceção da fase de pupa (Silva et al. 1994). Fato semelhante foi observado para C. raimundoi no presente estudo.

Indivíduos da espécie $C$. carnea tiveram seu tempo de desenvolvimento das fases imaturas prolongado, e a viabilidade dos ovos, consumo e habilidade de busca pela presa foram reduzidos em função do aumento no número de gerações utilizadas na experimentação (Jones et al. 1978).

Agradecimentos. Agradeço à Pos-doc Prof ${ }^{\text {a }}$. Dra ${ }^{\mathrm{a}}$. Adriana Coletto Morales pela revisão e sugestões feitas neste artigo.

\section{REFERÊNCIAS}

Andrewartha, H. G. \& L. C. Birch, 1954. Weather: Temperature, p.129171. In: H. G. Andrewartha \& L. C. Birch. (eds.). The Distribution and Abundance of Animals. London, The University of Chicago Press, $782 \mathrm{p}$.

Auad, A. M.; S. Freitas \& L. R. Barbosa. 2003. Potencial de alimentação de Chrysoperla externa (Hagen, 1861) (Neuroptera, Chrysopidae) em diferentes densidades de Uroleucon ambrosiae (Thomas, 1878) (Hemiptera, Aphididae). Revista Brasileira de Entomologia 47: $15-18$.

Canard, M. \& M. M. Principi. 1984. Development of Chrysopidae, p. 57-75. In: M. Canard; Y. Semeria \& T. R. New (eds). Biology of Chrysopidae. Netherlands, Dr W. Junk Publishers, 293 p.

ESTAT: sistema para análises estatísticas. Versão 2. 1992. Jaboticabal: FCAV.
Figueira, L. K.; C. F. Carvalho \& B. Souza. 2000. Biologia e exigências térmicas de Chrysoperla externa (Hagen, 1861) (Neuroptera: Chrysopidae) alimentada com ovos de Alabama argillacea (Hübner, 1818) (Lepidoptera: Noctuidae). Ciência e Agrotecnologia 24: 319-326.

Fonseca, A. R.; C. F. Carvalho \& B. Souza. 2001. Capacidade predatória e aspectos biológicos das fases imaturas de Chrysoperla externa (Hagen, 1861) (Neuroptera: Chrysopidae) alimentada com Schizaphis graminum (Rondani, 1852) (Hemiptera: Aphididae) em diferentes temperaturas. Ciências e Agrotecnologia 25: 251263.

Freitas, S. 2002. O uso de crisopídeos no controle biológico de pragas, p. 209-224. In: J. R. P. Parra; P. S. M Botelho; B. S. CorrêaFerreira \& J. M. S. Bento (eds.). Controle biológico no Brasil: parasitóides e predadores. São Paulo, Editora Manole, 609 p.

Haddad, M. L. \& J. R. P. Parra. 1984. Métodos para estimar os limites térmicos e a faixa ótima de desenvolvimento das diferentes fases do ciclo evolutivo de insetos. Piracicaba, Fundação de Estudos Agrários "Luiz de Queiroz", 12 p.

Jones, S. L.; R. E. Kinzer; D. L. Bull; J. R. Ables \& R. L. Ridgway. 1978. Deterioration fo Chrysopa carnea in mass culture. Annals of the Entomological Society of America 71: 160-162.

López-Arroyo, J. I.; C. A. Tauber \& M. J. Tauber. 2000. Storage of lacewing eggs: post-storage hatching and quality of subsequent larvae and adults. Biological Control 18: 165-171.

Murata, A. T.; A. C. Caetano; S. A. Bortoli \& C. H. Brito. 2006 Capacidade de consumo de Chrysoperla externa (Hagen, 1861) (Neuroptera: Chrysopidae) em diferentes presas. Caatinga 19: 304-309.

Parra, J. R. P. 2001. Previsão de produção, armazenamento e intercâmbio de insetos, p. 115-120. In: J. R. P. Parra. Técnicas de criação de insetos para programas de controle biológico. Piracicaba, ESALQ/ FEALQ, 134 p.

Pessoa, L. G. A.; S. Freitas \& E. S. Loureiro. 2004a. Efeito da variação da temperatura sobre o desenvolvimento embrionário e larval de Ceraeochrysa cincta (Hagen) (Neuroptera: Chrysopidae). Arquivos do Instituto Biológico 71: 1-719.

Pessoa, L. G. A.; M. V. Leite; S. Freitas \& G. C. Garbin. 2004b. Efeito da variação da temperatura sobre o desenvolvimento embrionário e pós-embrionário de Ceraeochrysa paraguaria (Navás) (Neuroptera: Chrysopidae). Arquivos do Instituto Biológico 71: 473-476.

Osman, M. Z. \& B. J. Selman. 1993. Storage of Chrysoperla carnea Steph (Neuroptera: Chrysopidae) eggs and pupae. Journal of Applied Entomology 115: 420-424.

Saini, E. D. 1997. Almacenaje de Chrysoperla externa (Hagen) (Neuroptera: Chrysopidae). Revista de Investigaciones Agropecuarias 28: 69-72.

Silva, R. L. X.; C. F. Carvalho \& M. Venzon. 1994. Aspectos biológicos das fases imaturas de Ceraeochrysa cubana (Hagen, 1861) (Neuroptera: Chrysopidae), em quatro gerações sucessivas em laboratório. Ciência e Prática 18: 13-17.

Tauber, M. J.; C. A. Tauber; K. M. Daane \& K. S. Hagen. 2000. Commercialization of predators: recent lessons from green lacewings (Neuroptera: Chrysopidae: Chyrsoperla). American Entomologist 46: $26-38$.

Tulisalo, U. 1984. Mass rearing techniques, p. 213-220. In: M. Canard; Y. Semeria \& T. R. New (eds). Biology of Chrysopidae. Netherlands, W. Junk Publishers, 293 p. 